\title{
Internal and effective stresses in nanocrystalline electrodeposited $\mathrm{Ni}$
}

\author{
S. Van Petegem, S. Brandstetter, and H. Van Swygenhoven ${ }^{\text {a) }}$ \\ Spallation Neutron Source Division, Paul Scherrer Institut, CH-5232 Villigen PSI, Switzerland \\ J-L. Martin \\ Institute of Physics of Complex Matter, Ecole Polytechnique Fédérale, CH-1015 Lausanne, Switzerland
}

(Received 1 February 2006; accepted 28 June 2006; published online 14 August 2006)

\begin{abstract}
Stress reduction experiments performed during tensile deformation of nanocrystalline electrodeposited Ni demonstrate high values for the effective and the internal stress as compared to coarse grained metals and evidence the existence of a negative creep. The results are interpreted in terms of a thermally activated dislocation mechanism where propagation is hindered by pinning at grain boundaries. (C) 2006 American Institute of Physics. [DOI: 10.1063/1.2336726]
\end{abstract}

It is well known that the stress necessary to deform a nanocrystalline metal is much higher than the stress needed to deform its coarse grained counterpart. ${ }^{1-3}$ Furthermore nanocrystalline metals exhibit an increased strain rate sensitivity. ${ }^{4,5}$ Direct visualization techniques such as transmission electron microscopy (TEM) failed to demonstrate that at small grain sizes the dominant deformation mechanism can still be considered as dislocation based., ${ }^{2,6}$ However, indirect methods such as in situ x-ray diffraction experiments suggest the presence of inhomogeneous strain during deformation, which are recoverable upon unloading at room temperature but unrecoverable when the experiment is performed at lower temperatures. ${ }^{7,8}$ Molecular dynamics (MD) suggests a slip mechanism where dislocations are emitted from grain boundaries (GBs), traveling through the entire grain, being absorbed in the opposite grain boundaries. Currently under discussion is whether the rate limiting process has to be related to the nucleation and/or propagation of dislocations. ${ }^{9,10}$ Measurements of activation volume using stress relaxation or strain rate experiments ${ }^{11}$ evidenced small values of the order of $10-20 b^{3}$, but the atomistic picture does not allow us to favor or exclude nucleation or propagation. $^{10}$

In a simplified picture the shear stress $\tau$ applied to make a crystal deform can be decomposed into an effective (thermal) stress $\tau^{*}$ and an internal stress $\tau_{i}$, usually found to be athermal, ${ }^{12}$

$$
\tau=\tau_{i}+\tau^{*}
$$

In coarse grained crystals, the internal stress results from long-range elastic interactions of mobile dislocations with the substructure and exhibits long-wavelength spatial fluctuations. The effective stress is related to short-range interactions with localized obstacles and exhibits short-range fluctuations. In other words, $\tau_{i}$ is that part of the applied stress needed to overcome long-range stress fields, whereas in combination with thermal fluctuations $\tau^{*}$ is needed to overcome the short-range obstacles.

It is possible to separate experimentally $\tau^{*}$ and $\tau_{i}$ by means of strain dip tests, ${ }^{13-18}$ where for different stress drop magnitudes, $\Delta \tau$, the subsequent initial creep rate $\dot{\gamma}_{p}$ is measured and observed to be positive, zero or eventually negative. The $\Delta \tau$ value that corresponds to a zero initial creep

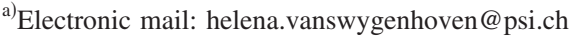

rate equals $\tau^{*}{ }^{13}$ This letter reports for the first time strain dip tests for nanocrystalline metals during constant strain rate tensile tests.

The experiments are performed at room temperature on nanocrystalline Ni synthesized by electrodeposition (ED-Ni), with a mean grain size of $30 \mathrm{~nm}$ as determined by both transmission electron microscopy and conventional x-ray diffraction. ${ }^{7}$ Mechanical testing is performed on $3 \mathrm{~mm}$ long dogbone-shaped samples. The strain is measured using a high resolution digital camera in combination with image recognition software, as described in Ref. 19.

The samples are loaded up to a certain applied stress level using a constant strain rate of $10^{-4} / \mathrm{s}$. Then the stress is dropped very rapidly (strain rate $>10^{-2} / \mathrm{s}$ ) by an amount $\Delta \tau$ followed by a short period of creep during which the plastic strain $\gamma_{p}$ is measured as function of time. It is assumed that the internal stress does not change during the stress drop, i.e., there is no significant change in the microstructure. The initial creep rate is determined by a linear fit of the first $10 \mathrm{~s}$ of creep. The dip tests were performed at three different points along the stress/strain curve between $2 \%$ and $4 \%$ true strains. For each different stress drop $\Delta \tau$ a different sample is used, all samples originating from the same batch and being treated in a similar way. The Taylor factor is used for extracting the shear stress $\tau$ from the applied normal stress.

Figure 1 shows the plastic strain as function of time for five different stress drops all performed at a true strain of $3 \%$. The corresponding creep rate as function of $\Delta \tau$ is shown

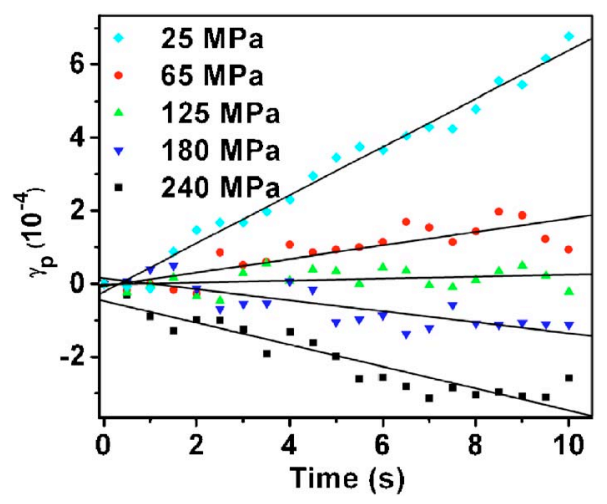

FIG. 1. (Color online) Creep curves after stress reduction at 3\% true strain (true stress of $1.8 \mathrm{GPa}$ ). The corresponding stress drops are given in the legend (full lines represent the linear fits). 


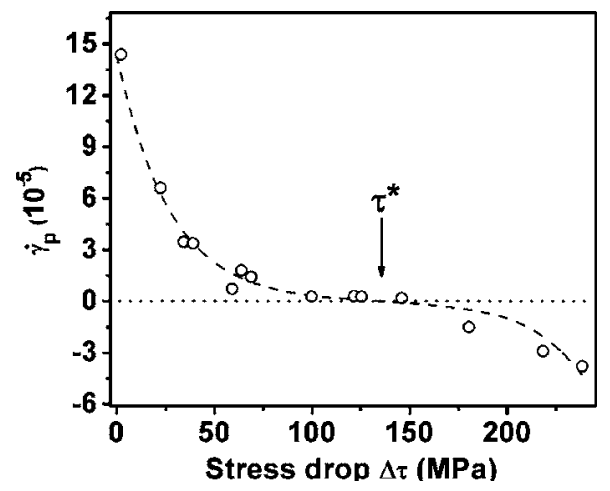

FIG. 2. Creep rate as function of stress drop $\Delta \tau$ for the data presented in Fig. 1. The dashed line represents the best fit obtained using Eq. (2).

in Fig. 2. As suggested by Milička, ${ }^{15} \tau^{*}$ can be found by fitting the creep rate versus stress drop by the following function:

$$
\dot{\gamma}_{p}=A \sinh \left[V\left(\tau^{*}-\Delta \tau\right) / k T\right],
$$

where $A$ is a temperature dependent coefficient including the mobile dislocation density, $V$ the activation volume, $T$ the absolute temperature and $k$ the Boltzmann constant. This equation is dictated by the thermal activation of dislocation motion. ${ }^{20}$ Figure 3 shows the internal and the effective normal stresses as a function of strain.

The above route for the determination of the effective stress [Eq. (2)] also allows calculation of the activation volume. However, it can also be derived considering two data points on Fig. 2 according to the following relationship, valid for small stress reductions: ${ }^{18}$

$$
\dot{\gamma}_{p 1} / \dot{\gamma}_{p 2}=\exp \left[V\left(\Delta \tau_{2}-\Delta \tau_{1}\right) / k T\right] .
$$

Equation (3) has the advantage over Eq. (2) that $\tau^{*}$ does not need to be known explicitly. We find an activation volume of $10 b^{3}$ (where $b$ is the Burgers vector of a dislocation). This is in good agreement with the results obtained in Ref. 11 for ED-Ni by stress relaxation experiments.

The above results can be summarized as follows.

(1) Both effective and internal stresses as well as their ratio $\left(\tau^{*} / \tau_{i} \approx 0.3\right)$ are large as compared to what is measured in single crystal fcc metals (values of 3 and $44 \mathrm{MPa}$ are reported for, respectively, the effective and internal shear

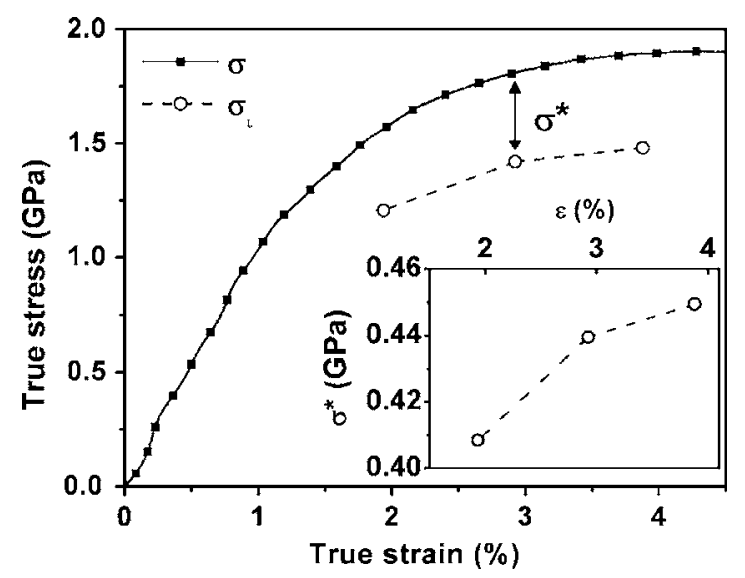

FIG. 3. Macroscopic stress/strain curve together with the internal stress. The inset shows the effective stress as a function of strain.

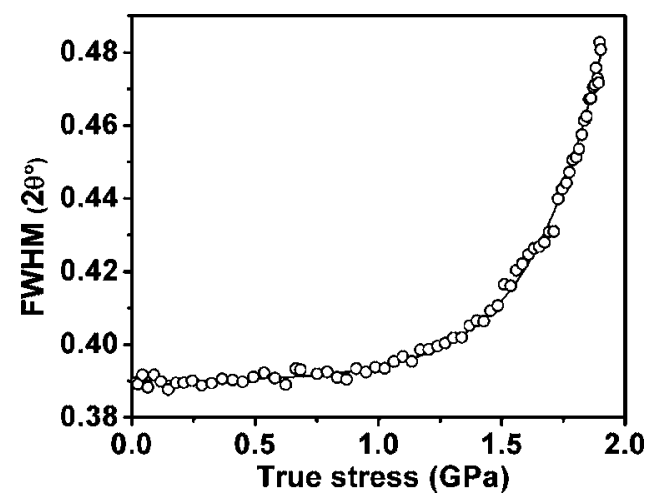

FIG. 4. Full width at half maximum of the (311) diffraction peak as a function of the true stress.

stresses for $\mathrm{Cu}$ at an $18.5 \%$ shear strain-athermal stage II of work hardening ${ }^{17}$ ). This is in agreement with the results obtained using strain rate jump tests. ${ }^{5}$ The high value of the effective stress in ED-Ni suggests a strong thermally activated mechanism in agreement with the small activation volume. The large value of the internal stress implies that the force for dislocation propagation is high compared to the usual force needed for a dislocation to cross a forest.

(2) The effective stress increases with strain, at least above a true stress of $1500 \mathrm{MPa}$, corresponding with the stress where plastic deformation mechanisms come fully at work. The latter is demonstrated in Fig. 4 by the strong increase in (311) peak width above $1500 \mathrm{MPa}$, a result obtained using in situ $\mathrm{x}$-ray diffraction profile analysis. ${ }^{5}$

(3) ED-Ni shows a negative creep for the larger stress drops. Negative creep rates have also been reported for $\mathrm{Ge}$ and $\mathrm{Cu}-7.5$ at. $\% \mathrm{Al}$ single crystals, ${ }^{17}$ unlike for a $\mathrm{Cu}$ single crystal.

A remarkable fact is that the curve of Fig. 2 exhibits the same features as those of polycrystalline metals where the dislocation velocity is controlled by their interaction with pinning points such as forest dislocations, impurities, or impurity clusters. This suggests that pinning points also control dislocation propagation in $\mathrm{ED}-\mathrm{Ni}$, the nature of which is going to be defined. The above mentioned results together with our current knowledge on dislocation mechanisms in nanocrystalline metals hint for a picture schematically presented in Fig. 5.

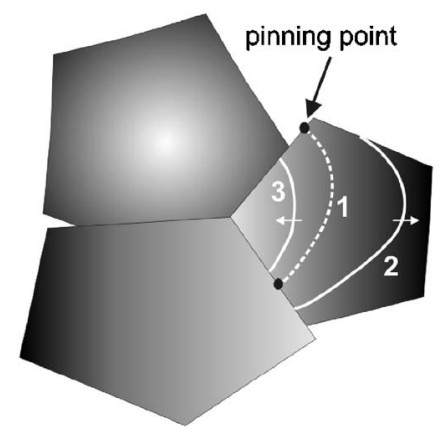

FIG. 5. Schematic representation of dislocation propagation in a grain during a stress dip test. The gray values represent fluctuations of the internal stress. 


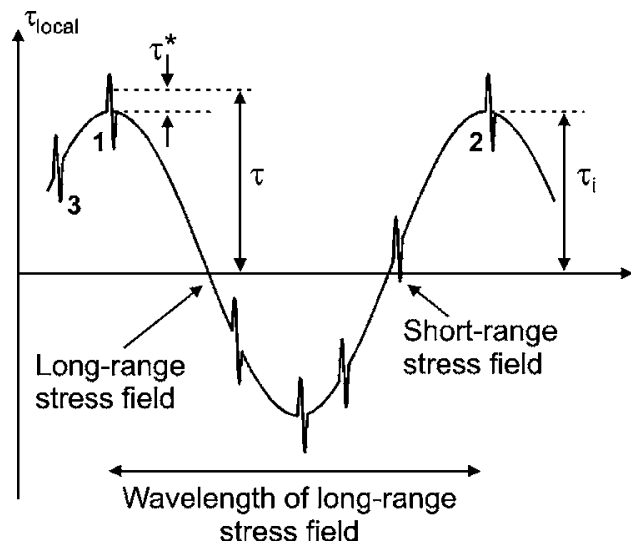

FIG. 6. Schematic representation of the stress fields experienced by dislocations along the slip plane.

In the as-prepared state, a nanocrystalline structure is characterized by a high density of interfaces containing intrinsic and extrinsic grain boundary dislocations and triple junctions, and nearly-defect-free grain interiors, in other words a very heterogeneous structure in terms of dislocation densities. This generates a heterogeneous distribution of internal stress (witnessed by strong contrast gradients observed in TEM pictures ${ }^{2}$ and a $0.4 \%$ root-mean-square strain in ED-Ni measured by $x$-ray diffraction ${ }^{7}$ ), schematically represented by the gray gradients in Fig. 5 .

On the moment of the stress drop, it can be assumed that several dislocations have been nucleated and are propagating. As suggested by MD simulations, a dislocation will be hindered on its way across the grain interior by the presence of GB ledges, acting as pinning points which have to be overcome by thermal activation. ${ }^{80}$ These pinning points represent the localized obstacles with short-range interactions. The total local stress field experienced by dislocations is given by the superposition of the long- and short-range stress fields ${ }^{21}$ as shown in Fig. 6.

The dislocations are held up most effectively against pinning points where the internal stress is opposite to the applied stress. Consider a dislocation bypassing obstacle (1) (in Figs. 5 and 6). When the stress drop is less than $\tau^{*}$ it will still propagate forward till position (2), producing a positive creep. However, when the stress drop exceeds $\tau^{*}$ the dislocations will run back and eventually stop at pinning point (3), producing a negative creep. In this picture, $\tau^{*}$ represents an average stress needed to get over a pinning point at constant strain rate. The fact that for ED-Ni significant negative creep rates are observed together with the reversibility in the peak broadening during in situ $\mathrm{x}$-ray diffraction upon unloading ${ }^{7}$ indicates that dislocations run easily back into
GBs. The increase in $\tau^{*}$ with strain points towards an increase of the pinning point density in the GBs, and the increase of $\tau_{i}$ towards changes in microstructure that are related with an increase in long-range stress fields. Such a picture could be in agreement with MD simulations; however, it cannot be confirmed due to restrictions in simulation time: indeed simulations demonstrate continuously changing GB structures and therefore ledge densities during dislocation nucleation and propagation, where extrinsic GB dislocations can be the signature of incomplete absorption, as such contributing to long-range stress fields. ${ }^{10}$

In summary strain dip tests evidence high values for the effective and the internal stress when compared to coarse grained structures and the existence of a negative creep. The results can be interpreted in terms of a dislocation mechanism where propagation is hindered by pinning at grain boundary ledges controlling the thermally activated dislocation mobility.

${ }^{1} \mathrm{~J}$. R. Weertman, in Nanostructured Materials: Processing, Properties, and Applications, edited by C. C. Koch (William Andrews, Norwich, NY, 2002), p. 397.

${ }^{2}$ K. S. Kumar, H. Van Swygenhoven, and S. Suresh, Acta Mater. 51, 5743 (2003).

${ }^{3}$ C. C. Koch, Scr. Mater. 49, 657 (2003).

${ }^{4}$ F. Dalla Torre, H. Van Swygenhoven, and M. Victoria, Acta Mater. 50, 3957 (2002).

${ }^{5}$ Y. M. Wang, A. V. Hamza, and E. Ma, Acta Mater. 54, 2715 (2006).

${ }^{6}$ R. C. Hugo, H. Kung, J. R. Weertman, R. Mitra, J. A. Knapp, and D. M. Folstaedt, Acta Mater. 51, 1937 (2003).

${ }^{7}$ Z. Budrovic, H. Van Swygenhoven, P. M. Derlet, S. Van Petegem, and B. Schmitt, Science 304, 273 (2004).

${ }^{8}$ S. Brandstetter, Z. Budrovic, S. Van Petegem, B. Schmitt, E. Stergar, P. M. Derlet, and H. Van Swygenhoven, Appl. Phys. Lett. 87, 231910 (2005).

${ }^{9}$ R. J. Asaro and S. Suresh, Acta Mater. 53, 3369 (2005).

${ }^{10}$ H. Van Swygenhoven, P. M. Derlet, and A. Frøseth, Acta Mater. 54, 1975 (2006).

${ }^{11}$ Y. M. Wang, A. V. Hamza, and E. Ma, Appl. Phys. Lett. 86, 241917 (2005).

${ }^{12}$ A. Seeger, J. Diehl, S. Mader, and H. Rebstock, Philos. Mag. 2, 323 (1957).

${ }^{13}$ G. B. Gibbs, Philos. Mag. 13, 317 (1966).

${ }^{14}$ W. Blum and A. Finkel, Acta Metall. 30, 1705 (1982).

${ }^{15}$ K. Milička, Acta Mater. 47, 1831 (1999).

${ }^{16}$ M. J. Mills, J. C. Gibeling, and W. D. Nix, Acta Metall. 33, 1503 (1985).

${ }^{17}$ T. Kruml, O. Coddet, G. Saada, and J. L. Martin, Philos. Mag. Lett. 83, 651 (2003).

${ }^{18}$ T. Kruml, O. Coddet, and J. L. Martin, Z. Metallkd. 96, 6 (2005).

${ }^{19}$ H. Van Swygenhoven, B. Schmitt, P. M. Derlet, S. Van Petegem, A. Cervellino, Z. Budrovic, S. Brandstetter, A. Bollhalder, and M. Schild, Rev. Sci. Instrum. 77, 013902 (2006).

${ }^{20}$ D. Caillard and J. L. Martin, in Thermally Activated Mechanisms in Crystal Plasticity, Pergamon Materials Series Vol. 8, edited by R. W. Cahn (Pergamon, New York, 2003), p. 227.

${ }^{21}$ H. Conrad, J. Met. 16, 582 (1964). 